\title{
Histological and immunohistochemical evaluation of sentinel lymph nodes in breast cancer at a tertiary hospital in the Western Cape, South Africa
}

\author{
A van Zyl, MB ChB; J W Schneider, MB ChB, MMed (Anatomical Pathology), FCPath \\ Division of Anatomical Pathology, Faculty of Medicine and Health Sciences, Stellenbosch University and National Health Laboratory Service, \\ Tygerberg Hospital, Cape Town, South Africa
}

Corresponding author: A van Zyl (adri@sun.ac.za)

\begin{abstract}
Background. Breast carcinoma remains the most prevalent cancer among women, with over 300000 deaths annually worldwide. Axillary lymph node status is essential for the clinical staging of breast carcinoma and remains the single most important predictor of disease-free survival in breast carcinoma.

Objective. To determine effective histological examination of sentinel lymph node (SLN) sections for the detection of metastatic breast carcinoma.

Methods. A prospective hospital-based study was done, including 20 patients with confirmed infiltrating breast carcinoma who underwent tumour excision or simple mastectomy as well as SLN biopsies. All the lymph nodes harvested were sectioned and embedded. Three sets of 15 consecutive serial sections were prepared from each case at one sitting, each measuring $3-5 \mu \mathrm{m}$ in thickness and mounted on separate slides. Each set of 15 consecutive sections was grouped into three levels, each comprising 5 serial sections. The first 4 sections were stained with haematoxylin and eosin (H\&E). The fifth section was stained for pancytokeratins, using MNF116.

Results. Twenty patients who met the inclusion criteria of this study underwent SLN biopsies and simple mastectomies or tumour excisions. Twelve SLNs of 11 patients contained metastatic carcinoma, all detected at level I, with one case requiring MNF116 immunohistochemistry staining, revealing metastatic carcinoma, measuring $0.08 \times 0.08 \mathrm{~mm}$ (micrometastases). The size of metastatic carcinoma ranged between $0.08 \times 0.08 \mathrm{~mm}$ (micrometastases) and $25 \times 15 \mathrm{~mm}$. Nine cases showed macrometastases, varying in size between $2 \times 3.5 \mathrm{~mm}$ and 25 $\times 15 \mathrm{~mm}$. Tumour sections of three patients with infiltrating carcinoma, of no specific type (NST), revealed lymphovascular invasion. The breast tumour sizes of these cases measured $40 \times 25 \mathrm{~mm}$ (1/1 node involved), $30 \times 20 \mathrm{~mm}(1 / 3$ nodes involved) and $15 \times 12 \mathrm{~mm}$ (1/1 node involved), respectively. Nine patients (19 nodes in total, mean 2.1, range $1-5$ ) did not have demonstrable metastatic disease in the 45 sections of levels I - IX, including MNF116 on every fifth section. Patients with negative SLNs varied in age between 29 and 68 years and had breast tumour sizes ranging between $10 \times 10 \mathrm{~mm}$ and $30 \times 30 \mathrm{~mm}$, respectively.

Conclusion. This study supports a conservative and cost-effective approach that comprises embedding of the entire SLN and the histopathological examination of four H\&E-stained sections, which will usually demonstrate metastatic carcinoma. In the event of absence of metastatic carcinoma, immunohistochemical staining for pancytokeratin will detect tumour cells in a small percentage of cases. Examination of additional H\&E- or pancytokeratin-stained sections is not cost effective. This finding can guide decisions pertaining to protocols for the histopathological assessment of SLN in breast carcinoma especially in resource-limited settings.
\end{abstract}

S Afr Med J 2016;106(5):528-530. DOI:10.7196/SAMJ.2016.v106i5.9909

Breast carcinoma remains the most prevalent cancer among women, with over 300000 deaths annually worldwide. ${ }^{[1]}$ According to the National Cancer Registry of South Africa (SA), the frequency of histologically diagnosed cases of breast carcinoma in SA for 2008 (latest available figures) is 5923 women and 146 men per annum.

Axillary lymph node status is essential for the clinical staging of breast carcinoma and remains the single most important predictor of disease-free survival in breast carcinoma. ${ }^{[1-3]}$ Only $20-30 \%$ of nodenegative patients will develop recurrence within 10 years, compared with about $70 \%$ of patients with axillary node involvement. ${ }^{[4,5]}$ It is postulated that the sentinel lymph node (SLN) is the first lymph node to receive lymphatic drainage from a tumour and is therefore the node most likely to contain metastatic disease. ${ }^{[6]} \mathrm{A}$ tumour-free SLN virtually excludes lymphatic involvement of the entire regional lymphatic basin. ${ }^{[4]}$

Tumour size is one of the most powerful predictors of tumour behaviour in breast cancer. ${ }^{[4]}$ The frequency of nodal metastases in patients with tumours smaller than $10 \mathrm{~mm}$ is $10-20 \%{ }^{[4]}$
SLN biopsy has emerged as a potential alternative to axillary dissection for staging of breast carcinoma. ${ }^{[6,7]}$ The technique is sensitive and specific to predict the axillary lymph node status. ${ }^{[6,7]}$ SLN biopsy without axillary dissection is beneficial because it may reduce the morbidity associated with axillary dissection. ${ }^{[4]}$ The reported falsenegative rates in the histological examination of SLNs range from $0 \%$ in smaller, single institution studies to $11.4 \%$ (range $0-28.6 \%$ ) in a large multi-institution study. ${ }^{[4]}$

Several recommendations pertaining to techniques for the histopathological assessment of an SLN have been published, but a standardised and internationally accepted protocol remains controversial. ${ }^{[6,7]}$ Without clear guidelines, sampling and preparation of histological slides may be inconsistent. The histological detection of micrometastases and single tumour cells requires skill and can be missed by an inexperienced pathologist. ${ }^{[3]}$ Immunohistochemistry (IHC) has improved the detection of micrometastases and single tumour cells..$^{[1,6]}$ Current acceptable standards of practice include intraoperative frozen sections of SLN for the detection of metastatic breast carcinoma. ${ }^{[6,7]}$ Intraoperative frozen section offers the advantage 
of an immediate result that can guide further intraoperative management and avoid the need for a second procedure at a later stage. However, intraoperative frozen section may be false-negative in up to $25 \%$ of cases because of suboptimal morphology and potential failure to demonstrate the subcapsular region of the lymph node.$^{[4]}$ Furthermore, micrometastases may be lost in the unexamined tissue subsequent to processing and embedding of the frozen tissue. ${ }^{[6]}$ In view of these limitations of intraoperative frozen sections, generally accepted practice requires at least three haematoxylin and eosin (H\&E)-stained tissue sections as well as at least one section for pancytokeratin IHC stain. ${ }^{[6,7]}$ This approach is feasible without incurring exorbitant costs. ${ }^{[6,7]}$ Significant resource implications of the increasing use of the SLN biopsy technique require cost-effective and accurate protocols for the histological examination of the SLN for detection of metastatic carcinoma. ${ }^{[6,7]}$

The American Society of Clinical Oncology (ASCO) guideline recommends that all submitted nodes be counted, measured and cut into perimeridianal (longitudinal) full cross-sections, no thicker than $2 \mathrm{~mm}$ and stained with $\mathrm{H} \& \mathrm{E}$ for histological assessment. ${ }^{[7]}$ Micrometastases will be detected in more instances with serial deeper levels than with fewer sections from the surface of the wax block only. IHC analysis with antibodies to cytokeratins (MNF116) facilitates the detection of isolated tumour deposits. ${ }^{[7]}$

The objective of this study was to determine effective histological examination of SLN sections for the detection of metastatic breast carcinoma in a tertiary hospital setting with limited resources. From these results, the goal was to establish a protocol for the histological examination of SLNs in breast carcinoma and enhance the management of patients through increased accuracy of detection of metastatic breast carcinoma in SLN biopsies. The outcome will facilitate optimisation of protocols for SLN assessment in resourcelimited environments.

\section{Methods}

A prospective hospital-based study was done at Tygerberg Hospital, Cape Town, SA, during the period 1 June 2014 - 31 August 2014. The study included 20 patients with confirmed infiltrating breast carcinoma who underwent tumour excision or simple mastectomy as well as SLN biopsies. Patients with known distant metastatic disease were excluded from the study. All participating patients gave informed consent for the use of their clinical data and tissue specimens in this study. The Health Research Ethics Committee of Stellenbosch University approved the study (Ref. No. 513/10/216).

All the lymph nodes harvested were sectioned, embedded and examined in the same way as discussed below. Each formalin-fixed SLN was sectioned at $2 \mathrm{~mm}$ intervals along its long axis and was embedded in its entirety with the sectioned surface face down. Thorough cleaning of instruments and work surface area minimised the risk of mechanical transportation of carcinoma fragments. All sections were processed and embedded in paraffin wax according to routine histopathological techniques. Three sets of 15 consecutive serial sections, each measuring $3-5 \mu \mathrm{m}$ in thickness and mounted on separate slides, were prepared from each case at one sitting, taking care to always use the same microtome in order not to lose any tissue in between sections.

Each set of 15 consecutive sections was grouped into three levels, each comprising five serial sections. The first four sections were stained with H\&E. The fifth section was stained for pancytokeratins, using MNF116. MNF116 labels epithelial tissues from simple glandular to stratified epithelium and is a useful tool for the identification of normal and neoplastic cells of epithelial origin. Monoclonal mouse antihuman cytokeratin, clone MNF116, Lot No. 69141 (Dakocytomation,
Denmark) is provided in liquid form as cell culture supernatant dialysed against $0.05 \mathrm{~mol} / \mathrm{L}$ Tris/ $\mathrm{HCl}, \mathrm{pH}$ 7.2, and contains $15 \mathrm{mmol} / \mathrm{L}$ $\mathrm{NaN}_{3}$. MNF116 is used at a dilution range of 1:100 and applied to formalin-fixed, paraffin-embedded sections of SLN, using 20 minutes' heat-induced epitope retrieval. IHC staining was performed using the Leica Bond autostainer (Leica Biosystems, SA).

In the absence of metastatic carcinoma in the first set of 15 sections, the procedure was repeated on the second set of 15 sections and, if still negative for metastatic carcinoma, also on the third set of 15 sections. No further sectioning was done after the third set (45 sections) in order to save costs and to avoid unnecessarily prolonged turnaround time of cases.

Metastatic carcinoma was distinguished from potential mimics including ectopic breast tissue, epithelial inclusions, displaced or transported tumour cells into lymph nodes and dendritic reticulum cells.

Metastatic disease was reported according to criteria as specified by the World Health Organization (WHO).$^{[8]}$ Specifically, all metastases $>2 \mathrm{~mm}$ in size were classified as macrometastases. A micrometastasis was diagnosed when one or more deposits of metastatic carcinoma were seen measuring $>0.2 \mathrm{~mm}$ in size, but none of which was $>2 \mathrm{~mm}$. Isolated tumour cells were reported when single or small clusters of tumour cells were identified measuring not $>0.2 \mathrm{~mm}$ in maximum dimension.

\section{Design and method pertaining to data analysis and statistics}

Data were collected in Excel (Microsoft, USA) and analysed using Statistica (version 11 of 2014, StatSoft, USA). Continuous data were described using means and standard deviations if normally distributed and medians and interquartile ranges if not normally distributed; $95 \%$ confidence intervals for the population mean were calculated. Categorical and ordinal data were described using frequency distributions. Data were presented graphically using either histograms or bar charts. A significance level of 5\% was applied throughout.

\section{Results}

Twenty patients who met the inclusion criteria of this study underwent SLN biopsies and simple mastectomies or tumour excisions. All the patients were female, with a mean age of 57 years (range $25-75$ years). Forty-four SLNs were available for analysis (mean 2.2 per patient, range 1 - 6).

Twelve SLNs of eleven patients, varying in age between 34 and 72 years, contained metastatic carcinoma, of which all were detected at level I. Ten patients had one positive lymph node each. One patient had two positive SLNs, the size of the metastatic tumour deposits measuring $5 \times 4 \mathrm{~mm}$ and $2 \times 2 \mathrm{~mm}$.

Nine cases showed macrometastases, varying in size between $2 \times 3.5 \mathrm{~mm}$ and $25 \times 15 \mathrm{~mm}$. One case showed micrometastases, measuring $0.08 \times 0.08 \mathrm{~mm}$ in size, detected by MNF116 at level I, $1 / 1$ node involved by tumour (infiltrating carcinoma, of no specific type (NST), 47-year-old with breast tumour size of $20 \times 20 \mathrm{~mm}$ ) and one case showed isolated tumour cells, $1 / 1$ node involved by tumour (infiltrating carcinoma, NST, 68-year-old with breast tumour size of $40 \times 25 \mathrm{~mm})$.

Eleven patients had metastatic carcinoma, with primary breast tumour sizes ranging between $40 \times 25 \mathrm{~mm}$ and $10 \times 10 \mathrm{~mm}$. Ten of the patients underwent simple mastectomy and SLN biopsy and one patient underwent tumour excision and SLN biopsy. Six patients had infiltrating carcinoma, NST, and there was one patient each with the following tumour types: colloid carcinoma, medullary-like, classic lobular, tubulo-lobular and infiltrating papillary carcinoma. 
Tumour sections of three patients with infiltrating carcinoma, NST, revealed lymphovascular invasion. The breast tumour sizes of these cases measured $40 \times 25 \mathrm{~mm}$ (1/1 node involved), $30 \times 20 \mathrm{~mm}$ $(1 / 3$ nodes $)$ and $15 \times 12 \mathrm{~mm}(1 / 1$ node $)$, respectively.

Nine patients (19 nodes in total, mean 2.1, range $1-5$ ) did not have demonstrable metastatic disease in the 45 sections of levels I - IX, including MNF116 on every fifth section. These patients with negative SLNs ranged in age between 29 and 68 years and had breast tumour sizes ranging between $30 \times 30 \mathrm{~mm}$ and $10 \times 10 \mathrm{~mm}$. Of these patients with negative SLNs, five patients underwent simple mastectomies and SLN biopsies and four patients underwent tumour excision and SLN biopsy. Five patients had infiltrating carcinoma, NST, and one patient had infiltrating duct carcinoma with apocrine differentiation.

Five of the 11 patients $(82 \%)$ with metastatic tumour received neo-adjuvant chemotherapy prior to SLN biopsy. Their nodal status ranged from $1 / 1,1 / 3$ and $1 / 4$ nodes involved by metastatic tumour. Six of the nine patients (66\%) without metastatic tumour received neo-adjuvant chemotherapy. Three had complete tumour response with nodal status of $0 / 4,0 / 2$ and $0 / 5$ nodes involved by metastatic tumour, respectively.

Statistical analysis of data confirmed that the cases with metastatic tumour did not share predictive characteristics. The smallest breast tumour (medullary-like type) that showed metastatic disease on H\&E staining was $10 \times 10 \mathrm{~mm}$ in size, whereas two tumours (infiltrating duct carcinoma, NST) which did not show metastatic disease, also measured $10 \times 10 \mathrm{~mm}$ in size each. One tumour (infiltrating duct carcinoma, NST) without metastatic disease, measured $30 \times 26 \mathrm{~mm}$ in size.

\section{Discussion}

SLN biopsy is rapidly emerging as an alternative to axillary lymph node dissection in staging the axilla in patients with early breast cancer. ${ }^{[7]}$ SLN biopsy has been incorporated into the ASCO guideline; however, the optimal histopathological work-up of SLNs is currently not standardised. ${ }^{[7]}$

Klevesath et al. ${ }^{[1]}$ prepared SLNs by cutting them in $2 \mathrm{~mm}$ slices longitudinally and found that 56/216 patients (25.9\%) showed metastatic tumour on H\&E only. A further 9 patients (4.2\%) had metastatic deposits detected by IHC, of whom 4 ( $2.0 \%$ of total, $44.4 \%$ of those detected by IHC) had micrometastatic disease, ranging from $0.2 \mathrm{~mm}$ to $1.0 \mathrm{~mm}$ in size. Similar to the findings of Klevesath et al. ${ }^{\left[{ }^{[1]}\right.}$ this study demonstrated that all the positive SLNs were detected at level I (4 H\&E stains and one MNF116 IHC stain). All the SLNs that were negative at level I remained negative in subsequent levels II - IX.

Weaver ${ }^{[6]}$ recommended that nodes be sectioned at $2 \mathrm{~mm}$ intervals and found that all metastases larger than $2.0 \mathrm{~mm}$ were detected by examination of one section from the surface of the block. They acknowledged that more comprehensive sampling would identify additional micrometastases and isolated tumour cells even though their prognostic significance is still unclear.

Lyman et al. ${ }^{[7]}$ recommended that nodes be cut into longitudinal slices no thicker than $2 \mathrm{~mm}$ and that at a minimum, full cross- sections of each SLN slice should be prepared and examined with H\&E staining. They stated that additional micrometastases were more likely to be detected with step sections at $200-500 \mu \mathrm{m}$ intervals than with superficial serial sections alone.

In this study, lymph nodes were sectioned at $2 \mathrm{~mm}$ intervals longitudinally and with serial sectioning, metastatic disease was detected within the first four H\&E-stained sections in 9/11 cases. The single case that required MNF116 IHC staining to confirm metastatic disease comprised infiltrating duct carcinoma, NST (metastatic tumour size: $0.8 \times 0.8 \mathrm{~mm}$; micrometastases).

Based on the results of this study, a cost-effective approach to the assessment of SLNs in breast carcinoma should include the histopathological evaluation of four H\&E-stained sections, which will detect the overwhelming majority of metastatic carcinoma. If metastatic carcinoma is absent, one IHC stain should be performed for pancytokeratins (such as MNF116), to aid in the detection of possible small foci of metastatic carcinoma. In addition to the high detection rate of metastatic carcinoma, this approach is always cost effective, especially in a resource-limited setting. During the course of this study one H\&E section cost R63.68 in the public sector and R85.80 in the private sector, while one MNF116 immunohistochemistry stain cost R384.86 in the public sector and R512.10 in the private sector, exclusive of VAT.

Owing to limited resources, this was a very small study, running over a 3-month period. Despite the small cohort of patients in this study, all specimens were handled optimally, without confounding technical or administrative errors.

\section{Conclusion}

The results obtained in this study justify a larger study with more patients to verify the findings and to determine potentially significant statistical influence on patient outcome.

Acknowledgements. We thank Prof. Justin Harvey (Stellenbosch University) for statistical assistance and the patients for participating in this study.

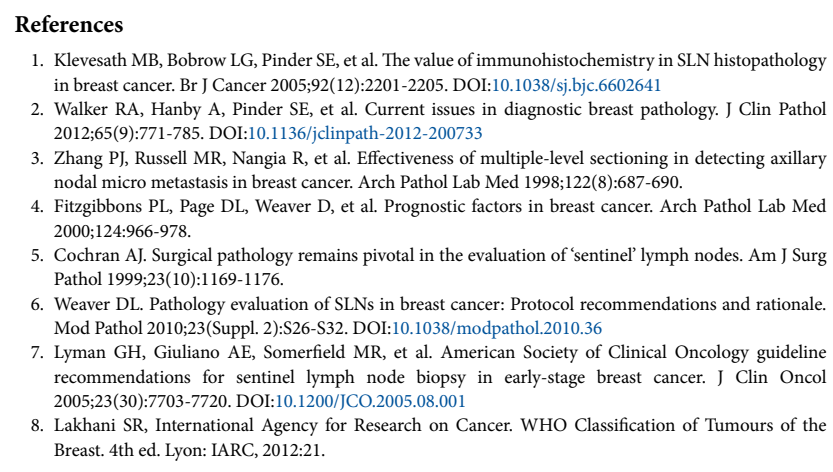

1. Klevesath MB, Bobrow LG, Pinder SE, et al. The value of immunohistochemistry in SLN histopathology in breast cancer. Br J Cancer 2005;92(12):2201-2205. DOI:10.1038/sj.bjc.6602641

2. Walker RA, Hanby A, Pinder SE, et al. Current issues in diagnostic breast pathology. J Clin Pathol 2012;65(9):771-785. DOI:10.1136/jclinpath-2012-200733

3. Zhang PJ, Russell MR, Nangia R, et al. Effectiveness of multiple-level sectioning in detecting axillary nodal micro metastasis in breast cancer. Arch Pathol Lab Med 1998;122(8):687-690.

4. Fitzgibbons PL, Page DL, Weaver D, et al. Prognostic factors in breast cancer. Arch Pathol Lab Med 2000;124:966-978.

5. Cochran AJ. Surgical pathology remains pivotal in the evaluation of 'sentinel' lymph nodes. Am J Surg Pathol 1999;23(10):1169-1176.

6. Weaver DL. Pathology evaluation of SLNs in breast cancer: Protocol recommendations and rationale. Weaver DL. Pathology evaluation of SLNs in breast cancer: Protocol reco
Mod Pathol 2010;23(Suppl. 2):S26-S32. DOI:10.1038/modpathol.2010.36

7. Lyman GH, Giuliano AE, Somerfield MR, et al. American Society of Clinical Oncology guideline . Lyman GH, Giuliano AE, Somerfield MR, et al. American Society of Clinical Oncology guideline
recommendations for sentinel lymph node biopsy in early-stage breast cancer. J Clin Oncol recommendations for sentinel lymph node biopsy in
2005;23(30):7703-7720. DOI:10.1200/JCO.2005.08.001

8. Lakhani SR, International Agency for Research on Cancer. WHO Classification of Tumours of the Breast. 4th ed. Lyon: IARC, 2012:21.

Accepted 2 March 2016. 